\title{
openheart Cardiovascular safety profile of taxanes and vinca alkaloids: 30 years FDA registry experience
}

\author{
Akshee Batra (D) , ${ }^{1}$ Brijesh Patel, ${ }^{2}$ Daniel Addison, ${ }^{3}$ Lauren A Baldassarre, ${ }^{4}$ \\ Nihar Desai, ${ }^{4}$ Neal Weintraub, ${ }^{5}$ Anita Deswal, ${ }^{6}$ Zeeshan Hussain, ${ }^{7}$ \\ Sherry-Ann Brown, ${ }^{8}$ Sarju Ganatra, ${ }^{9}$ Vivek Agarwala, ${ }^{10}$ Purvish M Parikh, ${ }^{11}$ \\ Michael Fradley, ${ }^{12}$ Arjun Ghosh, ${ }^{13}$ Avirup Guha (1) ${ }^{3,5,14}$
}

\begin{abstract}
- Additional supplemental material is published online only. To view, please visit the journal online (http://dx.doi.org/10. 1136/openhrt-2021-001849).

To cite: Batra A, Patel $B$, Addison D, et al. Cardiovascular safety profile of taxanes and vinca alkaloids: 30 years FDA registry experience. Open Heart 2021;8:e01849. doi:10.1136/ openhrt-2021-001849
\end{abstract}

$A G$ and $A G$ contributed equally.

Received 7 September 2021 Accepted 29 November 2021

Check for updates

(c) Author(s) (or their employer(s)) 2021. Re-use permitted under CC BY-NC. No commercial re-use. See rights and permissions. Published by BMJ.

For numbered affiliations see end of article.

Correspondence to Dr Avirup Guha; avirup.guha@ gmail.com

\section{ABSTRACT}

Objective Antimicrotubular agents are among the most commonly used classes of chemotherapeutic agents, but the risk of cardiovascular adverse events (CVAEs) remains unclear. Our objective was to study the CVAEs associated with antimicrotubular agents.

Methods The Food and Drug Administration's Adverse Event Reporting System was used to study CVAEs in adults from 1990 to 2020. Reported single-agent (only taxane or vinca alkaloid) CVAEs were compared with combination therapy (with at least one of the four major cardiotoxic drugs: anthracycline, HER2Neu inhibitors, tyrosine kinase inhibitors and checkpoint inhibitors) using adjusted polytomous logistic regression.

Results Over 30 years, 134398 adverse events were reported, of which 18426 (13.4\%) were CVAEs, with $74.1 \%$ due to taxanes and $25.9 \%$ due to vinca alkaloids. In 30 years, there has been a reduction in the proportion of reported CVAEs for taxanes from $15 \%$ to $11.8 \%$ (CochranArmitage P-trends $<0.001$ ) with no significant change in the proportion of reported CVAEs for vinca alkaloids $(9.2 \%-11.7 \%$; P-trends $=0.06)$. The proportion of reported CVAEs was lower in both taxane and vinca alkaloid monotherapy versus combination therapy (reporting $\mathrm{OR}=0.50$ and 0.55 , respectively). Anthracyclines and HER2Neu inhibitor combinations with taxanes or vinca alkaloids primarily drove the higher burden of combination CVAEs. Hypertension requiring hospitalisation and heart failure was significantly lower in monotherapy versus combination antimicrotubular agent therapy.

Conclusions Antimicrotubular agents are associated with CVAEs, especially in combination chemotherapy regimens. Based on this study, we suggest routine cardiovascular assessment of patients with cancer before initiating antimicrotubular agents in combination therapy.

\section{INTRODUCTION}

Antimicrotubule agents have been a part of the pharmacopoeia of cancer therapy for decades. They are used widely to treat Hodgkin's and non-Hodgkin's lymphoma, breast cancer, Kaposi sarcoma, non-small cell lung cancer, ovarian cancer and prostate cancer. These agents inhibit crucial microtubule

\section{Key questions}

What is already known about this subject?

- Antimicrotubular agents (taxanes and vinca alkaloids) are commonly used for various cancers as first-line treatment with a very poorly defined cardiovascular safety profile.

What does this study add?

- This study compares cardiovascular adverse events (CVAEs) due to antimicrotubular agents alone compared with combination therapy with four known cardiotoxic agents (anthracycline, HER2Neu inhibitors, tyrosine kinase inhibitors, checkpoint inhibitors). Our study is the first to quantify the relative CVAE of the antimicrotubular agent alone $(11.8 \%$ of all adverse events) and in combination ( $15 \%$ of all adverse events). Combination therapy, especially with anthracycline and HER2Neu inhibitors, has far more CVAEs than monotherapy alone. Hypertension requiring hospitalisation and heart failure was the most common CVAEs.

How might this impact on clinical practice?

- There are no guidelines for cardiovascular monitoring antimicrotubular agents. This data should prompt the inclusion of these agents in monitoring guidelines, primarily when used in specific combination therapies.

dynamics, inducing cellular responses like deregulated chromosome formation, abnormal mitotic spindle, metaphase arrest and eventual cell death. ${ }^{1}$ Although quite effective in cancer treatment, these agents are known to cause neurological and haematological toxicity due to this mechanism of action. $^{2}$ However, the degree of risk of cardiovascular complications due to microtubule inhibitors is indeterminate due to the use of multidrug chemotherapy regimens and conflicting literature. ${ }^{3-5}$

The incidence of heart failure has been reported at $2.3 \%-8 \%$ in single-centre studies 
and clinical trials consisting of patients taking taxanebased therapy. ${ }^{3}$ The most common cardiovascular adverse events (CVAEs) associated with antimicrotubule treatment are arrhythmias, including asymptomatic bradycardia, ventricular tachycardia, ${ }^{4}$ atrial fibrillation ${ }^{6}$ and other conduction system abnormalities. Myocardial ischaemia has also been reported with these agents. However, the results have been confounded by the concomitant use of radiation therapy and multi-regimen chemotherapy. ${ }^{47}$ In our study, we use the Food and Drug Administration (FDA)'s Adverse Event Reporting System (FAERS) to study the hypothesis that antimicrotubule therapy is safer from the cardiovascular standpoint and the relative risk of CVAEs is higher in combination with known cardiotoxic agents.

\section{METHODS}

This study was performed using the Strengthening the Reporting of Observational Studies in Epidemiology guidelines. 8

\section{Data source}

The FAERS is an international post-marketing safety surveillance system maintained by the FDA. It contains adverse event reports reported voluntarily for all marketed drug and therapeutic biologic products. ${ }^{9-11}$ The informatic structure of the FAERS database abides by the international safety reporting guidance issued by the International Conference on Harmonisation (ICH E2B). Adverse events and medication errors are coded to terms in the Medical Dictionary for Regulatory Activities (MedDRA) terminology based on the broad Standardised MedDRA Query. ${ }^{12}$ The database is publicly available, ${ }^{13}$ and as such, this study does not require ethics committee approval and informed consent and therefore complies with the Declaration of Helsinki. No patients were asked for input in the creation of this article.

\section{Data collection}

The FAERS registry was studied from 1 January 1990 to 6 September 2020, for adverse events reported for FDA-approved antimicrotubule agents, including taxanes and vinca alkaloids. Taxanes included paclitaxel (Taxol, Onxol, Abraxane, Nov-Onxol, Paclitaxel Novaplus), docetaxel (Docefrez, Taxotere) and cabazitaxel (Jevtana). Vinca-alkaloids included vincristine (Velban, Alkaban-AQ, Velbe, Velsar), vinblastine (Vincosid, Marqibo, Oncovin, Onkovin, Vincasar PFS, Vincrex, Vincrisul, Leurocristine) and vinorelbine (Navelbine). Reports that did not specify patient age or sex were excluded. Drug manufacturers, distributors and license holders are legally bound to actively collect and report all adverse effects related to their products. The FAERS event report form for each patient consists of case identification number, suspected medication, the reason for use, adverse reactions, nature of the event (ie, serious vs nonserious), outcomes (hospitalisation, death and other outcomes), event date, the pharmaceutical company, reporter (eg, healthcare professional, consumer, a pharmaceutical company or unknown), concomitant medications and country of the event. ${ }^{10} 1114$ Age was tiered into $18-39,40-59,60-79$ and $\geq 80$ years.

\section{Outcome}

Categories of specific CVAEs studied included atrial fibrillation, pericardial disease/effusion, myocardial infarction, ischaemic stroke, heart failure, venous thromboembolism (VTE) and hypertension requiring hospitalisation. All cardiovascular events included above and other minor CVAEs of QT prolongation, ventricular fibrillation and peripheral artery disease. These events are defined in online supplemental table 1 . The combination with four major concomitants and cardiotoxic drugs-anthracyclines, HER2Neu inhibitors, tyrosine kinase inhibitors and immune checkpoint inhibitors were compared with reported single-agent CVAEs.

\section{Statistics}

To determine the types and characteristics of CVAEs associated with antimicrotubule agents, the trend of CVAEs among all reported events for taxanes and vinca alkaloids from 1990 to 2020 was evaluated. Trends of reported cardiovascular events from 2010 to 2020 were also stratified by age and gender. Trends were analysed using the Cochran-Armitage test of trends. Reported single-agent (only taxane or vinca alkaloid) CVAEs were compared with combination therapy (with at least one of the four major cardiotoxic drugs: anthracycline, HER2Neu inhibitors, tyrosine kinase inhibitors and checkpoint inhibitors). The proportion of patients with reported CVAEs among all reported events was evaluated using adjusted polytomous logistic regression in single-agent versus combination therapy from 2010 to 2020 . Sensitivity analysis was conducted using the three most common CVAEs using subgroups of adverse events reported from the USA and European countries (France, Germany and Italy).

\section{RESULTS}

Over 30 years, 137398 adverse events (median age $=60$ years; $\mathrm{IQR}=50-68 ; 63.8 \%$ women) were reported with antimicrotubular agents. Among all reported adverse events, 29.3\% were for patients with breast cancer and $13.6 \%$ for patients with lymphoma, as the most frequently reported cancers. Overall, 18426 (13.4\%) CVAEs were reported. The reported CVAEs were in individuals with a median age of $64(\mathrm{IQR}=55-71)$ years and with a lower proportion of women compared with non-CVAEs $(58.4 \%$ vs $68.3 \%, \mathrm{p}<0.0001)$. The proportions of class-specific CVAEs included $74.1 \%$ due to taxanes (paclitaxel, docetaxel and cabazitaxel) and $25.9 \%$ due to vinca alkaloids (vinblastine, vincristine and vinorelbine) when considering all CVAEs. More than $40 \%$ of CVAEs were reported after 2014. Specific 
details regarding age distribution, reporting source, the indication of prescription of the antimicrotubular agent, the proportion of co-reported medications from the four classes of co-reported medications considered (anthracycline, HER2Neu inhibitor, tyrosine kinase inhibitor and checkpoint inhibitor) and proportion of the specific type of CVAE are presented in table 1.

The trend of antimicrotubular related CVAEs (1990-2020)

Among all reported adverse events, taxane-related CVAEs have gone down from $\sim 15 \%$ of all adverse events in the

Table 1 Characteristics of cardiovascular adverse event reporting on microtubular agents from 1990 to 2020

\begin{tabular}{|c|c|c|c|}
\hline Characteristic & $\begin{array}{l}\text { Taxanes } \\
(\mathrm{n}=13654)\end{array}$ & $\begin{array}{l}\text { Vinca alkaloids } \\
(\mathrm{n}=4772)\end{array}$ & $P$ value \\
\hline Age, $n(\%)$ & & & $<0.001$ \\
\hline $18-39$ years & $449(3.3)$ & $616(12.9)$ & \\
\hline $40-59$ years & $4274(31.3)$ & $1232(25.8)$ & \\
\hline $60-79$ years & $8287(60.7)$ & $2661(55.8)$ & \\
\hline$\geq 80$ years & $644(4.7)$ & $263(5.5)$ & \\
\hline Gender, n (\%) & & & $<0.001$ \\
\hline Male & 5036 (37.3) & 2521 (54.1) & \\
\hline Female & $8469(62.7)$ & $2138(45.9)$ & \\
\hline Year of reporting, $n$ (\%) & & & 0.0002 \\
\hline Before 2000 & $1098(8.0)$ & $473(9.9)$ & \\
\hline 2000-2009 & $3639(26.7)$ & $1208(25.3)$ & \\
\hline 2010-2014 & $2949(21.6)$ & $1073(22.5)$ & \\
\hline After 2014 & $5968(43.7)$ & 2018 (42.3) & \\
\hline Reporting source, $n(\%)$ & & & 0.01 \\
\hline Consumer & $54(0.4)$ & $37(0.8)$ & \\
\hline Pharmaceutical company & $11872(87.0)$ & $4114(86.2)$ & \\
\hline Healthcare professional & $851(6.2)$ & $301(6.3)$ & \\
\hline Other & $877(6.4)$ & $320(6.7)$ & \\
\hline \multicolumn{4}{|l|}{ Cardiovascular disease, $\mathrm{n}(\%)^{*}$} \\
\hline Hypertension requiring hospitalisation & $3415(25.0)$ & $961(20.1)$ & $<0.001$ \\
\hline Heart failure & $2312(16.9)$ & $1157(24.3)$ & $<0.001$ \\
\hline Atrial fibrillation & $1461(10.7)$ & $641(13.4)$ & $<0.001$ \\
\hline Pericardial disease/effusion & $129(0.9)$ & $57(1.2)$ & 0.14 \\
\hline Venous thromboembolism & 2927 (21.4) & $1092(22.9)$ & 0.04 \\
\hline Myocardial infarction & $1519(11.1)$ & $607(12.7)$ & 0.003 \\
\hline Ischaemic stroke & $1039(7.6)$ & $380(8.0)$ & 0.43 \\
\hline \multicolumn{4}{|l|}{ Indication for treatment, $\mathrm{n}(\%)$} \\
\hline Breast cancer & $3554(26.0)$ & $190(4.0)$ & $<0.001$ \\
\hline Lung cancer & $2632(19.3)$ & $414(8.7)$ & $<0.001$ \\
\hline Prostate cancer & $786(5.8)$ & $16(0.3)$ & $<0.001$ \\
\hline Lymphoma & $16(0.1)$ & $2455(51.5)$ & $<0.001$ \\
\hline Leukaemia & $62(0.5)$ & $394(8.3)$ & $<0.001$ \\
\hline Ovarian cancer & $1269(9.3)$ & $8(0.2)$ & \\
\hline \multicolumn{4}{|c|}{ Co-reported medications in the drug report, $\mathrm{n}(\%)$} \\
\hline Anthracycline & $1221(8.9)$ & $2296(48.1)$ & $<0.001$ \\
\hline HER2Neu inhibitors & $1691(12.4)$ & $97(2.0)$ & $<0.001$ \\
\hline Tyrosine kinase inhibitors & $120(0.9)$ & $151(3.2)$ & $<0.001$ \\
\hline Checkpoint inhibitors & $828(6.1)$ & $37(0.8)$ & $<0.001$ \\
\hline
\end{tabular}

*Proportion of all cardiovascular adverse events. 


\section{Percent reported Cardiovascular Events of all reported events}

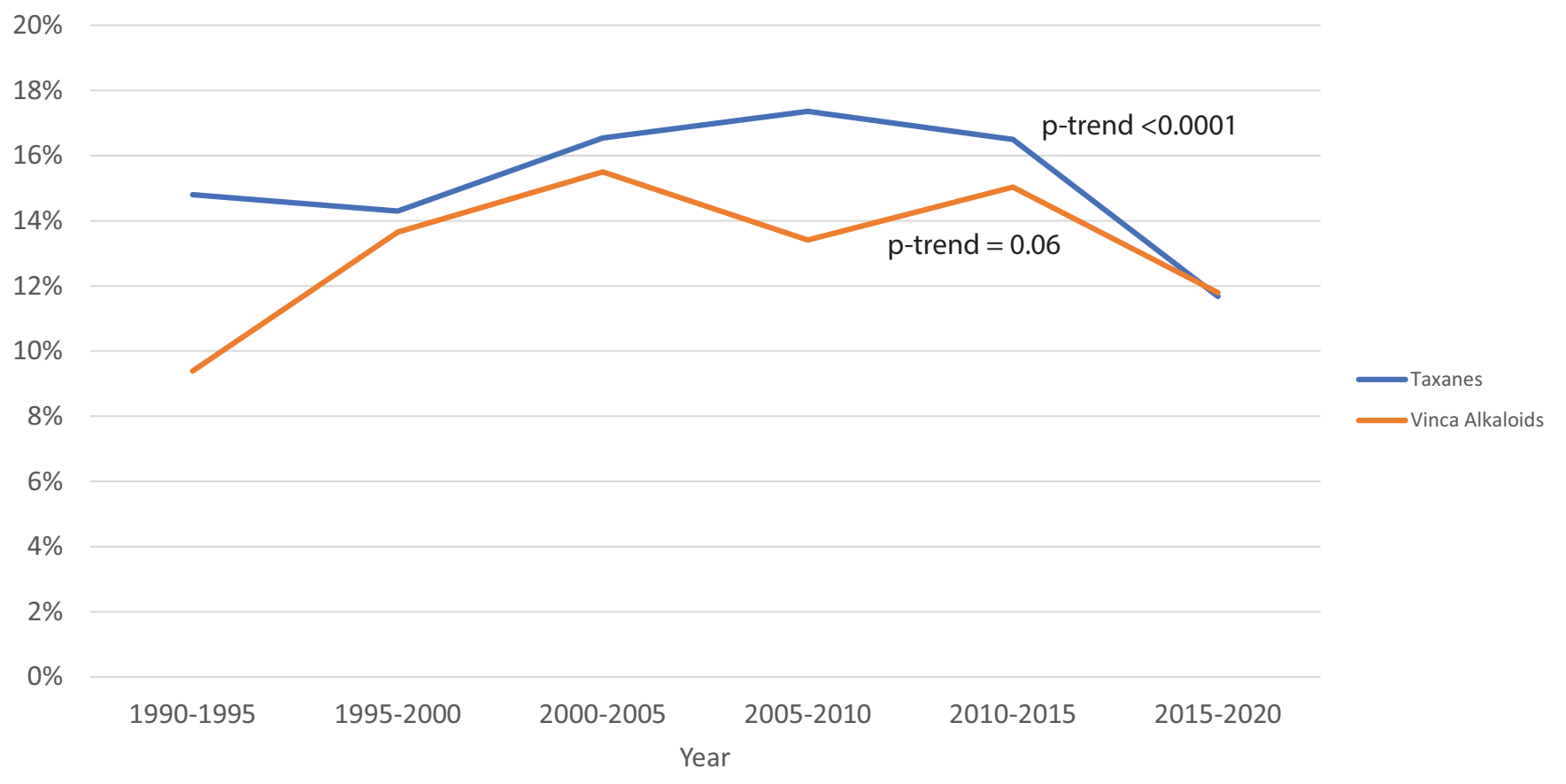

Figure 1 Trends of reported cardiovascular events among all reported events for taxanes and vinca alkaloids from 1990 to 2020.

1990 s to $11.8 \%$ in 2020 (Cochran-Armitage P-trends $<0.0001)$. The proportion of reported vinca alkaloidsrelated CVAEs increased from $9.2 \%$ to a high of $15.5 \%$ in 2003 to $11.7 \%$ in 2020 (Cochran-Armitage P-trends=0.06; figure 1). Since most of the adverse events were reported in the last 10 years, trends of the proportion of CVAEs stratified by age and gender were presented in online supplemental figure $1 \mathrm{~A}, \mathrm{~B}$. The proportion of taxane and vinca alkaloid-related CVAEs among all adverse events has decreased, with those above 65 years having a higher proportion of reported CVAEs compared with those below 65 years (online supplemental figure 1A, Cochran-Armitage P-trends for all four-curves $<0.001)$. For example, there was a reduction in the proportion of reported CVAEs among the reported adverse events with taxanes in those above 65 years from $22.4 \%$ in 2010 to $16.8 \%$ in 2020 (Cochran-Armitage P-trends <0.001). Gender stratified analyses demonstrate a decrease in the proportion of CVAEs in both men and women (CochranArmitage P-trends for all four-curves $<0.001$; online supplemental figure $1 \mathrm{~B}$ ).

\section{CVAEs as a single agent or in combination therapy (2010- 2020)}

The proportion of reported CVAEs was lower in taxanes (11.4\%) compared with taxane-based combination therapy $(17.9 \%$, reporting OR $(\mathrm{ROR})=0.5(95 \% \mathrm{CI}=0.35$ to 0.73$), \mathrm{p}=0.004)$. The likelihood of lower reported CVAE in taxanes was seen only in relation to combination of taxane with anthracyclines $(14.5 \%, \mathrm{ROR}=0.50$
(95\% CI $=0.33$ to 0.73$), \mathrm{p}=0.004)$ and taxanes with HER2Neu inhibitors $(16.8 \%, \mathrm{ROR}=0.55 \quad(95 \% \mathrm{CI}=0.35$ to 0.84$), \mathrm{p}=0.007)$. Similarly, the proportion of reported CVAEs was lower in vinca alkaloids-based monotherapy $(11.4 \%)$ versus combination therapy $(13.7 \%, \mathrm{ROR}=0.79$ (95\% CI $=0.73$ to 0.85 ), $\mathrm{p}<0.001$; table 2 ). There was no significant difference in proportion of reported CVAEs in taxanes or vinca alkaloid monotherapy and combination therapy with either a tyrosine kinase inhibitor or checkpoint inhibitor ( $\mathrm{p}$ for all pairwise comparison $>0.05$, table 2).

\section{Hypertension requiring hospitalisation (2010-2020)}

The most common CVAE related to taxanes was arterial hypertension requiring hospitalisation, with 2696 reported events among all reported CVAEs (30.2\%). Hypertension hospitalisation among all adverse events reported due to taxane monotherapy was reported at $3.4 \%$ compared with $5.3 \%$ among all adverse events reported with any taxane combination therapy (figure 2A). Hypertension requiring hospitalisation $(n=803)$ events was the third most common among reported CVAEs with vinca alkaloids. The specific proportion of hypertension events requiring hospitalisation among vinca alkaloid monotherapy and all four specific combination therapies of taxanes and vinca alkaloids are presented in figure 2A. The adjusted reporting OR was lower in both monotherapy arms compared with combination therapy (online supplemental table 2). 
Table 2 Cardiovascular adverse event reporting in combination with known cardiotoxic anticancer agents from 2010 to 2020

\begin{tabular}{|c|c|c|c|c|c|}
\hline Therapy & $\begin{array}{l}\text { \# CV/total } \\
\text { reported events }\end{array}$ & $\begin{array}{l}\% \text { of reported } \\
\text { events }\end{array}$ & Adjusted OR & $95 \% \mathbf{C l}$ & $P$ value \\
\hline Taxanes+any of the four agents & $2944 / 16458$ & 17.9 & Ref & - & - \\
\hline Taxanes & $6206 / 54332$ & 11.4 & 0.50 & 0.35 to 0.73 & 0.004 \\
\hline Taxanes+anthracyclines & $485 / 3341$ & 14.5 & Ref & - & - \\
\hline Taxanes & & & 0.55 & 0.35 to 0.84 & 0.007 \\
\hline Taxanes+HER2 Neu inhibitors & $1559 / 9273$ & 16.8 & Ref & - & - \\
\hline Taxanes & & & 0.39 & 0.26 to 0.58 & $<0.001$ \\
\hline Taxanes+tyrosine kinase inhibitors & $69 / 655$ & 10.5 & Ref & - & - \\
\hline Taxanes & & & 3.71 & 0.87 to 15.9 & 0.08 \\
\hline Taxanes+checkpoint inhibitor & $831 / 3189$ & 26.1 & Ref & - & - \\
\hline Taxanes & & & 0.53 & 0.22 to 1.26 & 0.15 \\
\hline Vinca alkaloids+any of the four agents & $2115 / 15445$ & 13.7 & Ref & - & - \\
\hline Vinca alkaloids & $1209 / 10646$ & 11.4 & 0.79 & 0.73 to 0.85 & $<0.001$ \\
\hline Vinca alkaloids+anthracyclines & $1759 / 12953$ & 13.6 & Ref & - & - \\
\hline Vinca alkaloids & & & 0.80 & 0.74 to 0.87 & $<0.001$ \\
\hline Vinca alkaloids+HER2 Neu inhibitors & $175 / 1139$ & 15.4 & Ref & - & - \\
\hline Vinca alkaloids & & & 0.58 & 0.49 to 0.70 & $<0.001$ \\
\hline Vinca alkaloids+tyrosine kinase inhibitors & $136 / 1032$ & 13.2 & Ref & - & - \\
\hline Vinca alkaloids & & & 0.86 & 0.71 to 1.05 & 0.14 \\
\hline Vinca alkaloids+checkpoint inhibitor & $45 / 321$ & 14.0 & Ref & - & - \\
\hline Vinca alkaloids & & & 0.86 & 0.62 to 1.19 & 0.36 \\
\hline
\end{tabular}

Total and cardiovascular adverse event reporting are shown. The OR for cardiovascular adverse event reporting (adjusted for age, gender, year of reporting, reporting source, need for hospitalisation, mortality) is shown as compared with any of the combinations.

$\mathrm{CV}$, cardiovascular.

\section{Cardiac adverse events (2010-2020)}

Heart failure was the most reported CVAE among vinca alkaloids ( $\mathrm{n}=805 ; 26.0 \%$ of all reported CVAEs). The reported heart failure was higher with combination therapy $(3.9 \%$ of all adverse events) compared with vinca alkaloid monotherapy $(2.5 \%$ of all adverse events; figure 2B). Among these reported heart failure events with vinca alkaloids, $27.0 \%$ died. Although heart failure was not as common among reported CVAEs for taxanes $(n=1478 ; 16.6 \%)$, the mortality was higher among adverse events of heart failure reported due to taxane monotherapy $(29.6 \%)$ compared with heart failure reported due to taxane combination therapy (19.6\%). Among all reported adverse events due to specific combination therapies, heart failure was highest with HER2Neu inhibitors among vinca combination $(4.0 \%)$ and anthracyclines
A

Hypertension requiring hospitalization

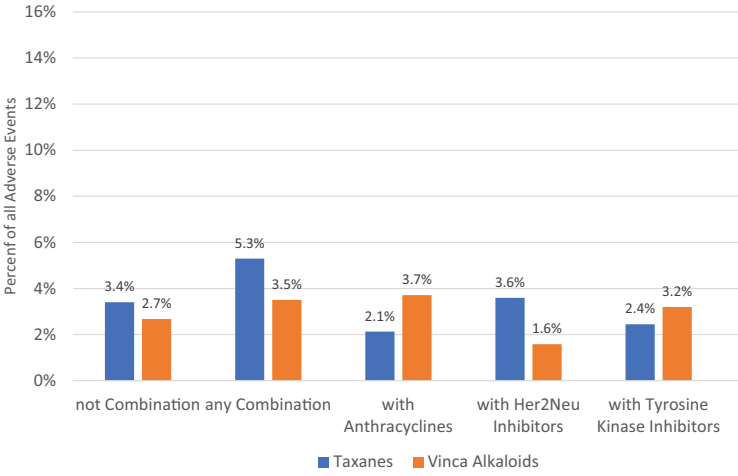

B

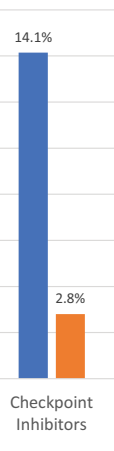

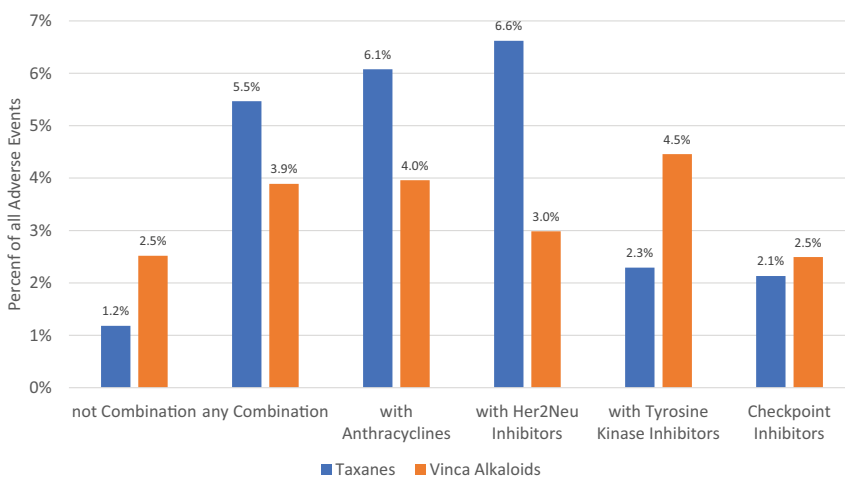

Figure 2 Proportion of patients requiring hospitalisation for $(A)$ hypertension and $(B)$ heart failure events among all reported events stratified based on the use of the type of combination with either taxanes or vinca alkaloids from 2010 to 2020 . Refer to tables 1 and 2 to see the total number of reported events to know the denominator for these events. 
among taxane combination therapies (6.6\%, figure $2 \mathrm{~B})$. The adjusted reporting OR of heart failure was lower in monotherapy compared with combination therapy in both taxanes and vinca alkaloids (online supplemental table 2).

There were 951 and 442 reports of atrial fibrillation among taxanes and vinca alkaloids, respectively $(10.7 \%$, and $14.3 \%$ of all CVAEs, respectively). However, the reporting of atrial fibrillation among all reported events was lower than 2\% (online supplemental figure 2A) among either antimicrotubular agents in monotherapy or combination therapy, except among combination of taxane with tyrosine kinase inhibitor (3.3\% of all adverse events), and vinca alkaloids with checkpoint inhibitors $(3.4 \%)$. Further, there was no difference in the adjusted reporting OR of atrial fibrillation in vinca alkaloid combination versus monotherapy (online supplemental table 2).

Over 30 years, pericardial events were reported in only 129 taxane-based and 57 on vinca alkaloid-based therapy (online supplemental figure 2B).

\section{Vascular adverse events (2010-2020)}

The second most common CVAE associated with taxane and vinca alkaloids was venous thromboembolism, with 1751 events $(19.6 \%)$ reported with taxanes and 712 events $(23.0 \%)$ in vinca alkaloid-related therapy. The proportion of venous thromboembolism from 2010 to 2020 remained below 4\% of all reported events in all monotherapy and combination therapy (online supplemental figure 3A) except the combination of vinca alkaloids and HER2Neu inhibitors $(6.8 \%)$ and checkpoint inhibitors with either vinca alkaloid or taxanes $(4.2 \%$ and $5.3 \%$, respectively; online supplemental figure $3 \mathrm{~A}$ ). There was no difference in the adjusted reporting OR of VTE event among monotherapy versus combination therapies of either vinca alkaloid or a taxane (online supplemental table 2).

Arterial vascular events that included myocardial infarction and stroke consisted of $15.7 \%$ and $16.6 \%$ of all CVAEs in taxanes and vinca alkaloids (breakdown presented in online supplemental table 2). The proportion of patients with myocardial infarction and stroke remained below $3 \%$ and $2 \%$ among all combinations of chemotherapies (online supplemental figure 3B,C). There was no difference in the adjusted reporting OR of all arterial vascular events in either monotherapy compared with combination therapy (online supplemental table 2).

\section{Other cardiac events (2010-2020)}

The other cardiac events were relatively rare. QT prolongation, ventricular fibrillation and peripheral vascular disease were reported in 5.9\%/6.3\%, 5.2\%/5.6\% and $1.4 \% / 4.5 \%$ of all CVAEs in taxanes and vinca alkaloids, respectively. The incidence of these events is infrequent among all adverse events in all combinations of chemotherapies $(<1 \%$, online supplemental figure 3D). Apart from peripheral vascular disease in taxanes, there was no difference in the adjusted reporting OR of QT prolongation and ventricular fibrillation events among monotherapy versus combination chemotherapies (online supplemental table 2).

\section{Sensitivity analysis (2010-2020)}

The reporting OR for hypertension requiring hospitalisation, heart failure, atrial fibrillation and venous thromboembolism in combination compared with monotherapy was recapitulated in the two different geographical areas (online supplemental table 3).

\section{DISCUSSION}

This study evaluated real-world data from the FAERS database to obtain information about CVAEs associated with antimicrotubular agents. Over 30 years, $13.4 \%$ of adverse events related to antimicrotubular agents were cardiovascular, and relative reporting has reduced over time. The overall proportion of reported CVAEs was lower with single-agent antimicrotubular therapy than combination therapy with anthracyclines or HER2Neu inhibitors. The most reported CVAE among taxanes and vinca alkaloids was hypertension requiring hospitalisation and heart failure, respectively. Among specific cardiovascular events, there was no difference in proportion and adjusted reporting OR of atrial fibrillation, venous or arterial vascular events, QT prolongation and ventricular fibrillation among either monotherapy or combination therapy.

Among those with taxane-related CVAEs, severe hypertension requiring hospitalisation was the most frequently reported event. In a study by Liu et al, among 710170 patients with cancer, hypertension was present in $10.8 \%$ of patients. ${ }^{15}$ This increasing prevalence of blood pressure abnormalities in patients with cancer suggests that therapy with antimicrotubule agents may only be one of the multiple risk factors leading to severe hypertension in patients with cancer. It is clear from prior data that HER2Neu inhibitors and tyrosine kinase inhibitors contribute to hypertension in patients with cancer. ${ }^{16}$

Heart failure was the most common CVAE reported among vinca alkaloids. Gehl et al demonstrated that doxorubicin and paclitaxel combination therapy resulted in congestive heart failure in $20 \%$ of patients, significantly higher than the incidence reported with single-agent antimicrotubular therapy. Paclitaxel and docetaxel have been reported to have a synergistic effect on cardiotoxicity with an anthracycline. ${ }^{17}$ The synergistic effect has been postulated due to increased doxorubicin metabolism to toxic metabolites. ${ }^{18}$ In our study, the proportion of heart failure events due to vinca alkaloids was $24.3 \%$ of all CVAEs, and around $49 \%$ of the events also involved the concomitant use of anthracyclines. Our study strengthens the notion of increased toxicity of antimicrotubular agents in combination; however, this needs to be tested prospectively while keeping in mind that anthracyclines, HER2Neu inhibitors, tyrosine kinase inhibitors and checkpoint inhibitors are known to cause heart failure. ${ }^{19-21}$ 
In the literature, cardiac ischaemia was reported in $5 \%$ of patients on paclitaxel therapy and $1.7 \%$ of patients treated with docetaxel. ${ }^{4}$ A study by Rowinsky et al demonstrated angina equivalents in 7 out of 140 patients treated with taxane-based therapy. ${ }^{4}$ In our study, we note arterial vascular events in both monotherapy and combination therapies with antimicrotubular agents, with no difference between combination and monotherapy. The true extent of the role of these agents in arterial vascular disease is challenging to determine as the results are complicated by radiation therapy and multiregimen chemotherapy.

Vinca alkaloids and taxanes are commonly used anticancer agents. However, formal cardiovascular risk assessment is difficult due to a dearth of literature assessing risk using contemporary treatment regimens and the lack of formal risk calculators for these agents. Previous work has mainly focused on qualitative reviews on the topic ${ }^{22}$ or pooled analysis with other agents focussing on risks associated with the management of a cancer type rather than specific qualitative risks with antimicrotubular agents. ${ }^{23}$ Our work details the cardiovascular use with solitary antimicrotubular therapy as well standard combination regimens widely used in clinical practice. Our data can be used as a surrogate for assessing individualised cardiovascular risk using these agents in contemporary cancer treatment regimens until they can be used to develop formal risk calculators and be incorporated into cardiovascular risk-assessment guidelines.

Multiple limitations must be acknowledged resulting from the use of data from a public registry. First, this retrospective study may be associated with reporting bias, including under-reporting, missing data or over-reporting of adverse events. Second, due to the absence of denominators, we could not discuss the incidence rate of each CVAEs. Third, we only considered four major concomitant cardiotoxic medications, while other combination therapy regimes were not analysed. Fourth, the rates of CVAEs in combination regimens with rates of CVAEs in other four drug classes alone without taxanes or vinca alkaloids have not been studied. Thus, we cannot comment on whether taxanes or vinca alkaloids add any CVAEs beyond the four drug classes when combined with them. Additionally, there has been a change in treatment regimens for cancer over the 30 years considered. Although we did not explicitly account for that change, the four combinations heavily reflect contemporary practice compared with decades prior. Fifth, clinical granularity such as knowledge of prior cardiovascular conditions, history, physical examination, laboratory values, ECG, echocardiogram, mortality adjusted cardiotoxic events or advanced cardiovascular workup was unavailable.

\section{CONCLUSION}

Antimicrotubular agent-related CVAEs are more common when prescribed with other cardiotoxic agents than when administered as sole agents. This report is the first attempt to create a knowledge base for antimicrotubular agent-related CVAEs to the best of our knowledge. Prospective studies are needed to determine the risk assessment scoring, preventive thresholds, monitoring and management guidelines for antimicrotubule agentsbased combination therapy.

\section{Author affiliations}

${ }^{1}$ Department of Medicine, University of Vermont Medical Center, Burlington, Vermont, USA

${ }^{2}$ Department of Cardiology, West Virginia University, Morgantown, West Virginia, USA ${ }^{3}$ Cardio-Oncology Program, Division of Cardiology, Department of Internal Medicine, The Ohio State University Wexner Medical Center, Columbus, Ohio, USA

${ }^{4}$ Department of Cardiology, Yale School of Medicine, New Haven, Connecticut, USA ${ }^{5}$ Cardio-Oncology Program, Division of Cardiology, Department of Internal Medicine, Augusta University Medical College of Georgia, Augusta, Georgia, USA

${ }^{6}$ Department of Cardiology, The University of Texas MD Anderson Cancer Center, Houston, Texas, USA

${ }^{7}$ Harrington Heart and Vascular Institute, University Hospitals, Cleveland, Ohio, USA

${ }^{8}$ Medical College of Wisconsin, Milwaukee, Wisconsin, USA

${ }^{9}$ Department of Cardiovascular Medicine, Lahey Clinic Medical Center, Burlington,

Massachusetts, USA

${ }^{10}$ Department of Medical Oncology, Narayana Superspeciality Hospital-Howrah,

Howrah, West Bengal, India

${ }^{11}$ Mumbai Oncocare Centers, Mumbai, Maharashtra, India

${ }^{12}$ Department of Medicine, University of Pennsylvania Perelman School of Medicine, Philadelphia, Pennsylvania, USA

${ }^{13}$ Barts and The London NHS Trust, London, UK

${ }^{14}$ Department of Internal Medicine, Case Western Reserve University, Cleveland, Ohio, USA

Twitter Akshee Batra @aksheebatra and Avirup Guha @avirupguha

Contributors AGu conceived the study concept, and the study design was by AGu and $A G$. AB and AGu contributed to conduct of study. AGu had full access to all the data in the study and took responsibility for the integrity of the data and the accuracy of the data analysis. AGu and AB drafted the first version of the manuscript. All authors provided critical revision of the manuscript for important intellectual content. AGu is also the guarantor for this study and accepts full responsibility for the work and/or the conduct of the study, had access to the data, and controlled the decision to publish.

Funding This work was supported in part by NIH grants P30 CA016058, K12CA133250 and K23-HL155890 (DA); and by American Heart Association-Robert Wood Johnson Foundation (Harold Amos) grant (DA). NLW is supported by NIH grants HL124097, HL126949, HL134354, AR070029 and AG064895. AGu and NLW are supported by American Heart Association-Strategically Focused Research Network Grant in Disparities in Cardio-Oncology (\#847740, \#863620).

Competing interests None declared.

Patient consent for publication Not applicable.

Ethics approval This study does not involve human participants.

Provenance and peer review Not commissioned; externally peer reviewed.

Data availability statement Data are available in a public, open access repository. The database is publicly available, and as such, this study does not require ethics committee approval and informed consent and therefore complies with the Declaration of Helsinki. No patients were asked for input in the creation of this article.

Open access This is an open access article distributed in accordance with the Creative Commons Attribution Non Commercial (CC BY-NC 4.0) license, which permits others to distribute, remix, adapt, build upon this work non-commercially, and license their derivative works on different terms, provided the original work is properly cited, appropriate credit is given, any changes made indicated, and the use is non-commercial. See: http://creativecommons.org/licenses/by-nc/4.0/.

\section{ORCID iDs}

Akshee Batra http://orcid.org/0000-0002-1994-1279

Avirup Guha http://orcid.org/0000-0003-0253-1174 


\section{REFERENCES}

1 Fanale D, Bronte G, Passiglia F, et al. Stabilizing versus destabilizing the microtubules: a double-edge sword for an effective cancer treatment option? Anal Cell Pathol 2015;2015:1-19.

2 Rowinsky EK, Donehower RC. The clinical pharmacology and use of antimicrotubule agents in cancer chemotherapeutics. Pharmacol Ther 1991;52:35-84.

3 Curigliano G, Mayer EL, Burstein HJ, et al. Cardiac toxicity from systemic cancer therapy: a comprehensive review. Prog Cardiovasc Dis 2010;53:94-104.

4 Rowinsky EK, McGuire WP, Guarnieri T, et al. Cardiac disturbances during the administration of taxol. J Clin Oncol 1991;9:1704-12.

5 Brugarolas A, Lacave AJ, Ribas A, et al. Vincristine (NSC 67574) in non-small cell bronchogenic carcinoma. Results of a phase II clinical study. Eur J Cancer 1978;14:501-5.

6 Arbuck SG, Strauss H, Rowinsky E, et al. A reassessment of cardiac toxicity associated with taxol. J Natl Cancer Inst Monogr 1993;15:117-30.

7 Vermorken JB, Remenar E, van Herpen C, et al. Cisplatin, fluorouracil, and docetaxel in unresectable head and neck cancer. $N$ Engl J Med 2007;357:1695-704.

8 von Elm E, Altman DG, Egger M, et al. The strengthening the reporting of observational studies in epidemiology (STROBE) statement: guidelines for reporting observational studies. Bull World Health Organ 2007;85:867-72.

9 Cortes J, Mauro M, Steegmann JL, et al. Cardiovascular and pulmonary adverse events in patients treated with Bcr-Abl inhibitors: data from the FDA adverse event reporting system. Am J Hematol 2015;90:E66-72.

10 Zhang KW, Reimers MA, Calaway AC, et al. Cardiovascular events in men with prostate cancer receiving hormone therapy: an analysis of the FDA adverse event reporting system (FAERS). J Urol 2021;206:613-22.

11 Guha A, Jain P, Fradley MG, et al. Cardiovascular adverse events associated with BRAF versus BRAF/MEK inhibitor: cross-sectional and longitudinal analysis using two large national registries. Cancer Med 2021;10:3862-72.
12 Lancellotti P, Suter TM, López-Fernández T, et al. Cardio-oncology services: rationale, organization, and implementation. Eur Heart $J$ 2019;40:1756-63.

13 FDA. Data from: FDA adverse event reporting system (FAERS) public Dashboard. Available: https://www.fda.gov/drugs/questions-andanswers-fdas-adverse-event-reporting-system-faers/fda-adverseevent-reporting-system-faers-public-dashboard [Accessed 21 Aug 2021].

14 Anand K, Ensor J, Trachtenberg B, et al. Osimertinib-induced cardiotoxicity: a retrospective review of the FDA adverse events reporting system (FAERS). JACC CardioOncol 2019;1:172-8.

15 Liu D, Ma Z, Yang J, et al. Prevalence and prognosis significance of cardiovascular disease in cancer patients: a population-based study. Aging 2019;11:7948-60.

16 Herrmann J. Vascular toxic effects of cancer therapies. Nat Rev Cardiol 2020;17:503-22.

17 Gehl J, Boesgaard M, Paaske T, et al. Combined doxorubicin and paclitaxel in advanced breast cancer: effective and cardiotoxic. Ann Oncol 1996;7:687-93.

18 Minotti G, Saponiero A, Licata S, et al. Paclitaxel and docetaxel enhance the metabolism of doxorubicin to toxic species in human myocardium. Clin Cancer Res 2001;7:1511-5.

19 Herrmann J. Adverse cardiac effects of cancer therapies: cardiotoxicity and arrhythmia. Nat Rev Cardiol 2020;17:474-502.

20 Jain P, Gutierrez Bugarin J, Guha A, et al. Cardiovascular adverse events are associated with usage of immune checkpoint inhibitors in real-world clinical data across the United States. ESMO Open 2021;6:100252

21 Dent SF, Morse A, Burnette S, et al. Cardiovascular toxicity of novel HER2-targeted therapies in the treatment of breast cancer. Curr Oncol Rep 2021;23:128.

22 Arora RD, Menezes RG. Vinca alkaloid toxicity. StatPearls. Treasure Island (FL): StatPearls Publishing Copyright (C) 2021, StatPearls Publishing LLC, 2021.

23 Clark RA, Marin TS, McCarthy AL, et al. Cardiotoxicity after cancer treatment: a process map of the patient treatment journey. Cardiooncology 2019;5:14. 